\title{
FORMULATION, DEVELOPMENT AND CHARACTERISATION OF NIMODIPINE LOADED SOLID LIPID NANOPARTICLES
}

\author{
REMYA P. N.1, DAMODHARAN N.2*
}

1,2Department of Pharmaceutics, SRM College of Pharmacy, SRM Institute of Science and Technology, Chennai, Tamilnadu, India Email: damodhan@srmist.edu.in

Received: 05 Feb 2020, Revised and Accepted: 30 Jul 2020

\begin{abstract}
Objective: The aim of the present study is to develop solid lipid nanoparticles (SLNs) of Nimodipine using hot homogenization followed by ultrasonication technique and to improve the dissolution characteristics of the drug.

Methods: The Nimodipine-loaded SLN was prepared using palmitic acid and stearic acid as a lipid matrix and Tween-80 as an emulsifier by a hot homogenization and ultra-sonication method. The physicochemical characteristics of SLN were investigated for entrapment efficiency, zeta potential, in vitro drug release, particle size analysis, Fourier transform infrared studies, scanning electron microscopy, and stability studies.

Results: The mean particle size, PDI, Zeta potential and entrapment efficiency of optimized Nimodipine SLN formulation of stearic acid was found to be $119.54 \mathrm{~nm}, 0.165,-17.60 \mathrm{mV}, 85 \%$ and for palmitic acid was found to be $132.54 \mathrm{~nm}, 0.155,-17.0 \mathrm{mV}$, $81 \%$ respectively. In vitro drug release studies indicated that after an initial burst release, SLN could provide prolonged release of Nimodipine. The selected SLNs have shown good stability for a period of $180 \mathrm{~d}$.
\end{abstract}

Conclusion: SLN formulations showed the best results in EE as well as in vitro drug release and therefore, these results indicate that SLN might be a promising delivery system to enhance the release of Nimodipine.

Keywords: Nimodipine, Solid lipid nanoparticles, Stearic acid, Palmitic acid, Tween-80

(C) 2020 The Authors. Published by Innovare Academic Sciences Pvt Ltd. This is an open access article under the CC BY license (http://creativecommons.org/licenses/by/4.0/) DOI: http://dx.doi.org/10.22159/ijap.2020v12i5.34342. Journal homepage: https://innovareacademics.in/journals/index.php/ijap

\section{INTRODUCTION}

Solid lipid nanoparticles introduced in 1991 represent an alternative carrier system to tradition colloidal carriers such as emulsions, liposomes and polymeric nanoparticles [1]. Nanoparticles made from solid lipids are attracting major attention as novel colloidal drug carriers for intravenous applications as they have been proposed as an alternative particulate carrier system. The system consists of spherical solid lipid particles in nanometre ranges, which are dispersed in water or in aqueous surfactant solutions. Generally, they are made of the solid hydrophobic core having a monolayer of phospholipid coating. The solid core contains the drug dissolved or dispersed in the solid high melting fat matrix. The hydrophobic chains of phospholipid are embedded in the fat matrix $[2,3]$. The solid lipid nanoparticles are sub-micron colloidal carriers from 50 $1000 \mathrm{~nm}$, which are composed of physiological lipid, dispersed in water or in aqueous surfactant solution. SLN offers unique properties such as small size, large surface area, high drug loading, and interaction of phases at the interface and attractive for their potential to improve the performance of pharmaceuticals [4]. Solid lipid nanoparticles (SLN) are at the forefront of the rapidly developing field of nanotechnology with several potential applications in drug delivery and research. Due to their unique sizedependent properties, lipid nanoparticles provide the possibility to develop newer therapeutics. The ability to incorporate drugs into nanocarriers offers a new prototype in drug delivery that could use for drug targeting $[5,6]$. Solid lipid nanoparticle is an efficient lipidbased drug delivery system which can improve the bioavailability of poorly water-soluble drugs. Nimodipine, a potent antihypertensive agent has been used in the treatment of hypertensive disorders. Nimodipine is a highly lipophilic drug and poorly water-soluble drug with the bioavailability of $13 \%$. The present work illustrates the development of an SLN formulation for Nimodipine with increased bioavailability [7, 8]. NSLNs were developed using Stearic acid, Palmitic acid as the lipid and Tween 80 as the surfactant by hot homogenization followed by an ultra-sonication method. NSLN's were characterized for Scanning and Transmission Electron Microscopy (SEM), Entrapment Efficiency (EE), Stability studies and Zeta Potential.
In vitro drug release studies were performed in $\mathrm{pH} 7.4$ phosphate buffer. SLN formulation F4 and A4 composed of Tween 80 as a surfactant and lower concentration of lipid matrix (1000 mg of stearic acid for F4 and $1000 \mathrm{mg}$ of Palmitic acid for A4) showed the best result in view of the entrapment efficiency as well as in vitro drug release; also the potential zeta studies showed that the formed particles were in Nano size and possess a negative surface charge.

\section{MATERIALS AND METHODS}

\section{Materials}

Nimodipine was purchased from Shreeji Pharma International, Vadodara, Gujarat and stearic acid is purchased form Lobachem Mumbai, Tween 80 and Palmitic acid were purchased from Sisco research Mumbai. The reagents used in this work all are analytical reagent grade.

Methods

\section{Selection of lipids}

The solubility of Nimodipine was determined by dissolving an excess amount of drug in 200 mg of lipids (Palmitic acid, Stearic acid Glyceryl monostearate, Glyceryl monooleate, cetyl palmitate, and cholesterol) in $5 \mathrm{ml}$ stoppered vial and then the mixture was mixed by using a vortex mixer. For equilibrium, the vials were then kept at $37 \pm 1.0{ }^{\circ} \mathrm{C}$ in an isothermal shaker (EXPO HI-TECH) for $72 \mathrm{~h}$. The drug was added in increments of $10 \mathrm{mg}$. The quantity after which no further solubilization of drug was taken endpoint of solubilization. The equilibrated samples were transferred to a centrifuged tube and rotated at $3500 \mathrm{rpm}$ for $15 \mathrm{~min}$. The supernatant layer obtained from centrifuge was filtered through a $0.45 \mu \mathrm{m}$ membrane filter. The concentration of drug was determined in each component by UV spectrophotometer.

\section{Selection of surfactants}

With the selected lipid, nanoparticles are prepared using different surfactants and were evaluated with respect to the particle size, PDI and entrapment efficiency. Selection of the surfactants was based on minimum particle size and PDI with maximum entrapment efficiency. 


\section{Preparation of SLNS}

The SLNs were produced using the modified high shear homogenization and ultra-sonication technique $[9,10]$. SLN was prepared by heating different concentrations of lipids (stearic acid and palmitic acid) and different concentrations of surfactants $(1 \%$ and $2 \%)$. The organic phase containing stearic acid and soya lecithin is added with the drug
Nimodipine and heated to a temperature of $70{ }^{\circ} \mathrm{C}$ until it dissolves completely and heating the aqueous phase containing surfactant tween 80 to the same temperature. The hot aqueous phase is added to the oil phase mixture and homogenized by using mechanical stirrer for $30 \mathrm{~min}$ to get a clear solution. It is then followed by probe sonication for $25 \mathrm{~min}$ $[11,12]$. This similar technique used for preparing SLN with palmitic acid. In table 1 SLNs formulation data was given.

Table 1: SLN formulations loaded with nimodipine

\begin{tabular}{|c|c|c|c|c|c|c|c|c|c|c|c|c|}
\hline Components & $\begin{array}{l}\text { F1 } \\
\text { (mg) }\end{array}$ & $\begin{array}{l}\text { F2 } \\
\text { (mg) }\end{array}$ & $\begin{array}{l}\text { F3 } \\
\text { (mg) }\end{array}$ & $\begin{array}{l}\text { F4 } \\
\text { (mg) }\end{array}$ & $\begin{array}{l}\text { F5 } \\
\text { (mg) }\end{array}$ & $\begin{array}{l}\text { F6 } \\
\text { (mg) }\end{array}$ & $\begin{array}{l}\text { A1 } \\
\text { (mg) }\end{array}$ & $\begin{array}{l}\text { A2 } \\
\text { (mg) }\end{array}$ & $\begin{array}{l}\text { A3 } \\
\text { (mg) }\end{array}$ & $\begin{array}{l}\text { A4 } \\
\text { (mg) }\end{array}$ & $\begin{array}{l}\text { A5 } \\
\text { (mg) }\end{array}$ & $\begin{array}{l}\text { A6 } \\
\text { (mg) }\end{array}$ \\
\hline $\begin{array}{l}\text { Drug } \\
\text { (Nimodipine) }\end{array}$ & 100 & 100 & 100 & 100 & 100 & 100 & 100 & 100 & 100 & 100 & 100 & 100 \\
\hline Stearic Acid & 1500 & 1500 & 1000 & 1000 & 500 & 500 & - & - & - & - & - & - \\
\hline Palmitic Acid & - & - & - & - & - & - & 1500 & 1500 & 1000 & 1000 & 500 & 500 \\
\hline Tween $80(\mathrm{ml})$ & 1 & 2 & 1 & 2 & 1 & 2 & 1 & 2 & 1 & 2 & 1 & 2 \\
\hline Soya lecithin & 500 & 500 & 500 & 500 & 500 & 500 & 500 & 500 & 500 & 500 & 500 & 500 \\
\hline $\begin{array}{l}\text { Distilled } \\
\text { water(ml) }\end{array}$ & 100 & 100 & 100 & 100 & 100 & 100 & 100 & 100 & 100 & 100 & 100 & 100 \\
\hline
\end{tabular}

\section{Characterization of nimodipine SLNs}

\section{Scanning electron microscopy (SEM) of nanoparticles}

The morphology of nanoparticles was investigated by SEM (SE S$3400 \mathrm{~N}$; HITACHI, Japan). Briefly, $10 \mathrm{mg}$ freeze-dried SLN was suspended in $1 \mathrm{ml}$ distilled water and $2 \mu \mathrm{l}$ of the suspension was placed on a glass surface. After oven-drying, the samples were coated with gold using an Ion Sputter and examined at an accelerating voltage of $20 \mathrm{kV}[13,14]$.

Particle shape and surface morphology by transmission electron microscopy (TEM)

Transmission electron microscopy (TEM) analysis of the prepared SLNs was carried out to study morphology like sphericity and aggregation. Sample was examined by TEM (H 100, Hitachi Ltd., Japan). Samples were stained with phosphotungstic acid (PTA, 2\%,5 min and excess PTA removed, spread on a grid and examined [15].

\section{Particle size and zeta potential determination}

The average particle size, polydispersity index, and zeta potential of the lipid particulate dispersions were performed using a zeta sizer (DTS Ver.5.10, Malvern Instruments). The dispersion was diluted to $1: 10 \mathrm{v} / \mathrm{v}$ with double distilled water (filtered through $0.45 \mu \mathrm{m}$ membrane filters) to determine that the light scattering intensity was within the instrument's sensitivity range $[16,17]$.

\section{Fourier transformed infrared (FTIR) spectroscopic analysis}

An FT-IR spectroscopy study has been carried out to check the compatibility between the drug (Nimodipine) and the lipid (SA and PA) separately, which are used for the preparation of nanoparticles. The FT-IR was performed for drug, lipids, and physical mixture of drug and lipid at a wavelength from 4000 to $400 \mathrm{~cm}-1$ [18].

\section{Entrapment efficiency of the Nimodipine drug}

The centrifugation method was used for measuring the entrapment efficiency of the SLN dispersion $[19,20]$. Supernatant liquid was collected by centrifuging the SLN dispersion (containing the $1 \mathrm{mg}$ of Nimodipine drug) at $2000 \mathrm{rpm}$ for one hour and then it was filtered to determine the free drug concentration. For the dilution of the sample, phosphate buffer saline with $\mathrm{pH} 7.4$ was used. The absorbance was determined at $236 \mathrm{~nm}$ with a UV spectrophotometer and the entrapment efficiency was calculated using the equation (1).

$$
\text { Entrapment efficiency of the drug }=\frac{\text { Total drug-free drug }}{\text { Total drug }} \times 100---(1)
$$

\section{In vitro dug release}

The in vitro drug release of different dispersions of SLNs were determined using the dialysis bag technique. SLN dispersion containing an equivalent of $5 \mathrm{mg}$ Nimodipine drug was transferred to the dialysis bag and the bag was sealed tightly. The sealed bag was suspended in the phosphate buffer saline and stirred with the help of a magnetic stirrer $[21,22]$. The conditions for the suspended solution were $\mathrm{pH} 7.4$ and the temperature of $37^{\circ} \mathrm{C} \pm 0.5^{\circ} \mathrm{C}$. After that, up to $12 \mathrm{~h}$, aliquots were withdrawn at different time periods and the quantity of drug released was calculated by using the spectrophotometric studies at $236 \mathrm{~nm}$.

\section{Stability studies}

The samples with high entrapment efficiencies were identified; for them Stability studies were conducted at two different temperatures of $4{ }^{\circ} \mathrm{C}$ and $25 \pm 2{ }^{\circ} \mathrm{C}$. The drug content was measured for every $15 \mathrm{~d}$ to note the changes in the prepared SLNs.

\section{RESULTS}

\section{Solubility studies}

The solubility of Nimodipine was determined by dissolving an excess amount of drug in $200 \mathrm{mg}$ of lipids (Palmitic acid, Stearic acid, Glyceryl monostearate, Glyceryl monooleate, Cetyl palmitate, and cholesterol) in $5 \mathrm{ml}$ stoppered vial and then the mixture was mixed by using vortex mixer. The supernatant layer obtained from centrifuge was filtered through a $0.45 \mu \mathrm{m}$ membrane filter. The concentration of the drug was determined at $236 \mathrm{~nm}$ for each component by UV spectrophotometer. The lipids Palmitic acid and Stearic acid given with higher solubility values when compared with other lipids and therefore, these lipids are used for further studies as shown in fig. 1.

Table 2: Solubilization of nimodipine in various lipids

\begin{tabular}{lll}
\hline S. No. & Lipids & Solubility(mg/gm) \\
\hline 1 & Glyceryl monostearate & $90 \pm 12$ \\
2 & Glyceryl monooleate & $70 \pm 10$ \\
3 & Cetyl palmitate & $60 \pm 11$ \\
4 & Stearic acid & $120 \pm 12$ \\
5 & Palmitic acid & $100 \pm 11$ \\
6 & Cholesterol & $80 \pm 11$ \\
\hline
\end{tabular}

mean. \pm SD $(n=3)$ 


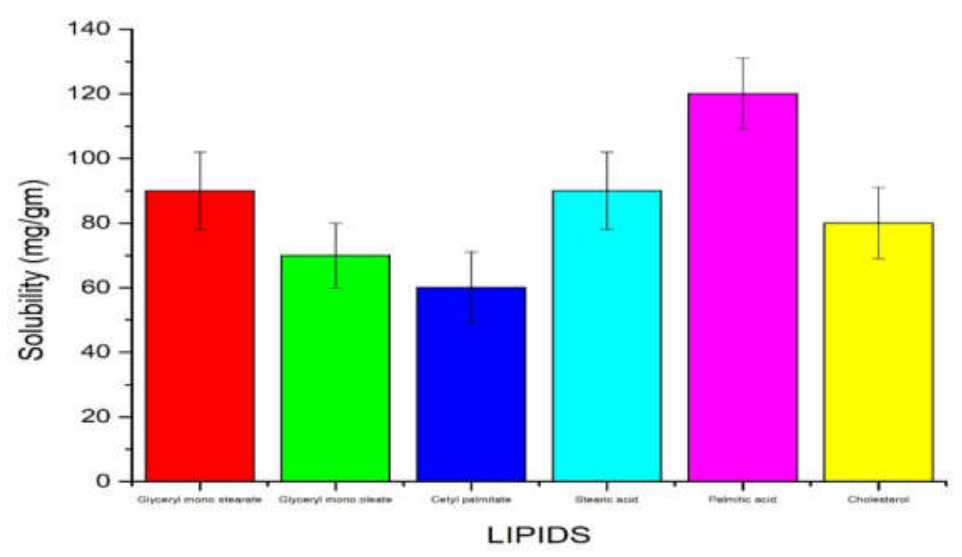

Fig. 1: Solubility profile of nimodipine in various lipids [mean. $\pm S D(n=3)]$

\section{Selection of surfactants}

The selected lipids (Palmitic acid and Stearic acid) are chosen for preparing nanoparticles using different grades of surfactant Tween
(20.40.80) and were evaluated with respect to the particle size, PDI and entrapment efficiency. The surfactant Tween 80 was selected based on its minimum particle size and PDI and with maximum entrapment efficiency.

Table 3: Selection of surfactants on the basis of particle size, PDI and entrapment efficiency for the drug nimodipine using lipid stearic acid and palmitic acid

\begin{tabular}{llllll}
\hline S. No. & Lipid(1 $\mathbf{g m} / \mathbf{m l})$ & Surfactant $\mathbf{( 1 \% )}$ & Particle size (nm) & PDI & Entrapment efficiency (\%) \\
\hline 1 & Stearic acid & Tween20 & $231.32 \pm 3.93$ & $0.174 \pm 0.066$ & $67.73 \pm 4.57$ \\
2 & Stearic acid & Tween40 & $301.41 \pm 3.96$ & $0.290 \pm 0.037$ & $56.35 \pm 5.67$ \\
3 & Stearic acid & Tween80 & $133.11 \pm 1.96$ & $0.169 \pm 0.068$ & $72.64 \pm 4.85$ \\
4 & Palmitic acid & Tween20 & $265.01 \pm 2.92$ & $0.222 \pm 0.057$ & $65.73 \pm 8.49$ \\
5 & Palmitic acid & Tween40 & $239.30 \pm 3.49$ & $0.196 \pm 0.053$ & $51.85 \pm 3.78$ \\
6 & Palmitic acid & Tween80 & $158.01 \pm 3.98$ & $0.122 \pm 0.052$ & $75.66 \pm 6.35$ \\
\hline
\end{tabular}

mean. \pm SD $(n=3)$

\section{Particle sizes and zeta potentials}

All the prepared formulations were analyzed to determine their particle size distributions and zeta potential values. The results obtained after particle size analysis are shown in table 4 . In all the formulations prepared using stearic acid had shown the particle sizes ranged from
119 to $138 \mathrm{~nm}$, and zeta potential- $17 \mathrm{mV}$ to- $37.6 \mathrm{mV}$ and polydispersity index was found to be between 0.162 to 0.265 (table 4) for formulations (F1 to F6). In all the formulations prepared using palmitic acid had shown the particle sizes ranged from 132 to $158 \mathrm{~nm}$, and zeta potential$17 \mathrm{mV}$ to-34.6mV and polydispersity index was found to be between 0.155 to 0.275 (table 4) for formulations (A1 to A6).

Table 4: Evaluation parameters of nimodipine loaded SLN formulations

\begin{tabular}{|c|c|c|c|c|c|c|c|}
\hline $\begin{array}{l}\text { Formulation } \\
\text { code }\end{array}$ & $\begin{array}{l}\text { Lipid } \\
\text { concentrati } \\
\text { on (mg) }\end{array}$ & $\begin{array}{l}\text { Surfactant } \\
\text { concentrati } \\
\text { on }(\%)\end{array}$ & $\begin{array}{l}\text { Entrapment } \\
\text { efficiency (\%) }\end{array}$ & $\begin{array}{l}\text { In vitro drug } \\
\text { release (\%) }\end{array}$ & $\begin{array}{l}\text { Particle } \\
\text { size (nm) }\end{array}$ & PDI & $\begin{array}{l}\text { Zeta potential } \\
(\mathrm{mV})\end{array}$ \\
\hline F1 & 1500 & 1 & $81.8 \pm 1.55$ & 76.1 & $138.71 \pm 2.95$ & $0.162 \pm 0.02$ & $-19.6 \pm 5.045$ \\
\hline $\mathrm{F} 2$ & 1500 & 2 & $83.8 \pm 1.35$ & 81.5 & $122.2 \pm 4.13$ & $0.174 \pm 0.055$ & $-18.4 \pm 6.055$ \\
\hline F3 & 1000 & 1 & $82.4 \pm 4.35$ & 80 & $133.11 \pm 3.93$ & $0.169 \pm 0.046$ & $-19.4 \pm 7.330$ \\
\hline $\mathrm{F} 4$ & 1000 & 2 & $85.8 \pm 2.45$ & 83 & $119.3 \pm 1.89$ & $0.165 \pm 0.044$ & $-17.6 \pm 4.065$ \\
\hline F5 & 500 & 1 & $80.3 \pm 2.45$ & 78 & $122.4 \pm 1.89$ & $0.265 \pm 0.034$ & $-27.6 \pm 4.065$ \\
\hline $\mathrm{F} 6$ & 500 & 2 & $81.2 \pm 2.45$ & 80 & $121.64 \pm 1.89$ & $0.205 \pm 0.064$ & $-37.6 \pm 4.065$ \\
\hline A1 & 1500 & 1 & $79.5 \pm 0.45$ & 80 & $133.9 \pm 3.96$ & $0.171 \pm 0.052$ & $-18.2 \pm 5.054$ \\
\hline $\mathrm{A} 2$ & 1500 & 2 & $80.9 \pm 0.55$ & 81 & $132.4 \pm 2.23$ & $0.170 \pm 0.056$ & $-18.5 \pm 8.050$ \\
\hline A3 & 1000 & 1 & $80.7 \pm 2.30$ & 83 & $135.0 \pm 3.93$ & $0.163 \pm 0.058$ & $-19.0 \pm 9.450$ \\
\hline $\mathrm{A} 4$ & 1000 & 2 & $81.8 \pm 3.45$ & 85 & $132.6 \pm 3.93$ & $0.155 \pm 0.052$ & $-17.0 \pm 7.055$ \\
\hline A5 & 500 & 1 & $76.8 \pm 3.45$ & 78 & $158.6 \pm 2.96$ & $0.245 \pm 0.052$ & $-34.0 \pm 7.055$ \\
\hline A6 & 500 & 2 & $79.8 \pm 3.45$ & 80 & $149.6 \pm 3.93$ & $0.275 \pm 0.022$ & $-28.0 \pm 7.055$ \\
\hline
\end{tabular}

mean. \pm SD $(n=3)$

\section{IR studies}

Sample and total mixture were studied by using IR spectral studies. The FT-IR spectrums of pure drug Nimodipine and formulation are shown in fig. 2, respectively. Samples retained their individual absorption characteristics, without undergoing any interaction with one another. From these studies, we can conclude that no untoward chemical reactions were observed. 


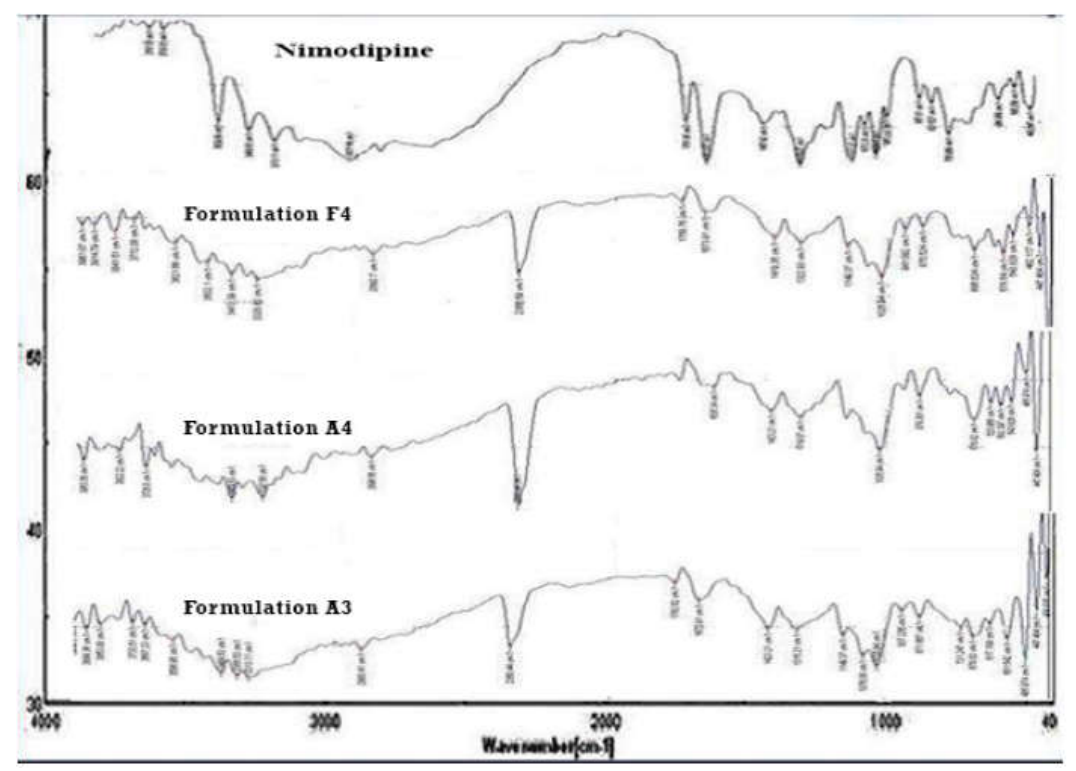

Fig. 2: Infrared spectrum of nimodipine and formulations

\section{Entrapment efficiency}

In table 4 the entrapment efficiency (responses) of the designed formulations was given. Entrapment efficiency was used to characterize the SLNs. The optimal encapsulation efficiency can decided by several factors like the concentration of the lipids and surfactants. From table 4, the entrapment efficiency was in the range of $81.2-85.8 \%$ for all formulations (F1toF6) and $76.8-81.8 \%$ for formulations (A1 to A6). This observation leads the statement that the decrease in the concentration of the lipid and increase in the concentration of the surfactant shows an increase in entrapment efficiency. F4 shows an entrapment efficiency of $85.8 \%$ and A4 shows $81.8 \%$ entrapment. Drug expulsion in SLN can occur when the lipid matrix transforms from high energy modifications characterized by the presence of many imperfections with forming a perfect crystal with no room for guest molecules. Palmitic acid-lipid molecules are chemically different, resulting in a structure with many imperfections to accommodate the drug and thus higher loading capacity. About $80 \%$ of entrapment efficiency is seen in all formulations. Due to the low content of lipid in formulation showed more drug release.

\section{In vitro drug release}

Percentage release of drug with different concentrations of lipid stearic acid and the lipid palmitic acid are shown in table 4 respectively. In vitro release profiles for Nimodipine for lipid stearic acid and the lipid palmitic acid were shown in fig. 3 and in fig. 4 . Fig. 3 shows that the in vitro studies of all the batches of stearic acid showed initial progress of $17 \%-30 \%$ of drug release for all the batches within 1hour and the $60 \%$ was released slowly over a period of $4 \mathrm{~h}$. The batches F1-F6 shows about $80 \%$ of the Nimodipine drug was released at the end of $12 \mathrm{~h}$. From the fig. 4 it was observed that A4 shows a drug release of $85 \%$ at the end of 12 hours and it is the highest compared to other lipid palmitic acid batches. All experiments were done in triplicate and values are expressed in means and standard deviation. In summary, an increase in drug release with increase in surfactants was observed.

\section{Morphological studies}

The SEM images for the SLNs loaded with nimodipine drugs were shown in fig. 5 and in fig. 6 . From the morphological studies, it is revealed that the SLNs were in spherical shape.

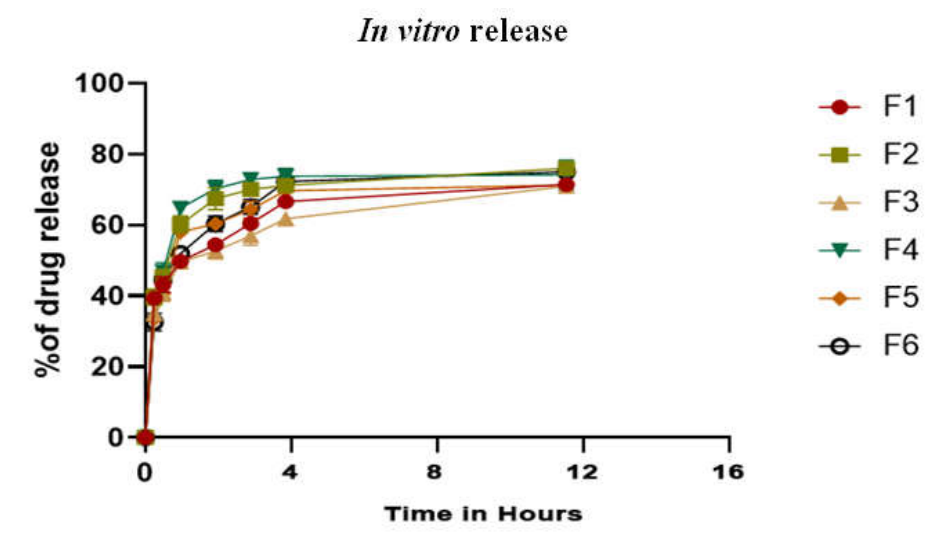

Fig. 3: In vitro release profile of nimodipine for different batches of drug-loaded with tween 80 and stearic acid [mean. $\pm S D(n=3)]$ 
In vitro release

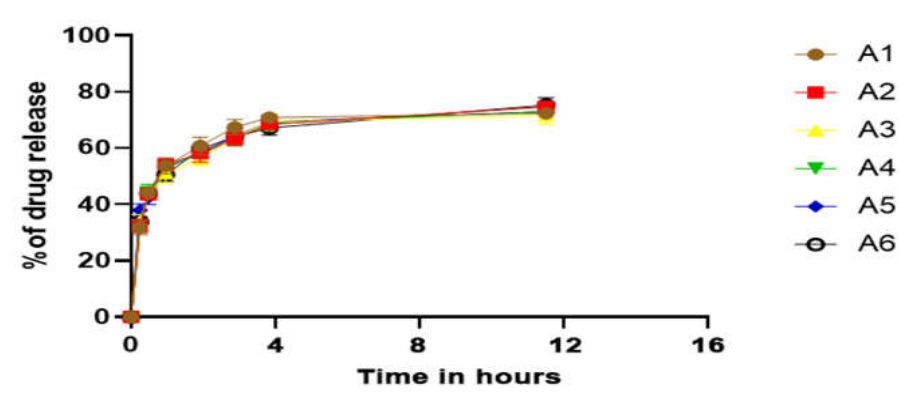

Fig. 4: In vitro release of Nimodipine for different batches of drug-loaded with Tween 80 and palmitic acid [mean. $\pm S D(n=3)]$

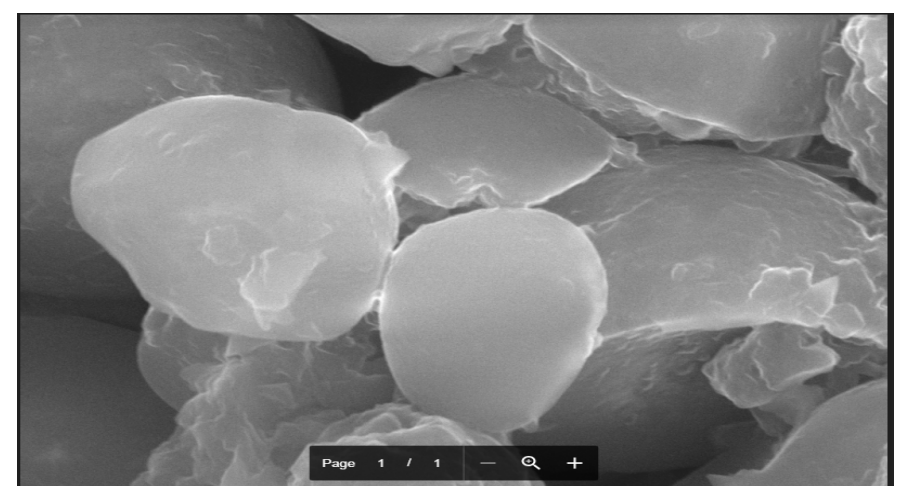

Fig. 5: Scanning electron microscopy of nimodipine SLN F4 (Containing tween 80, stearic acid and drug concentration-100 mg)

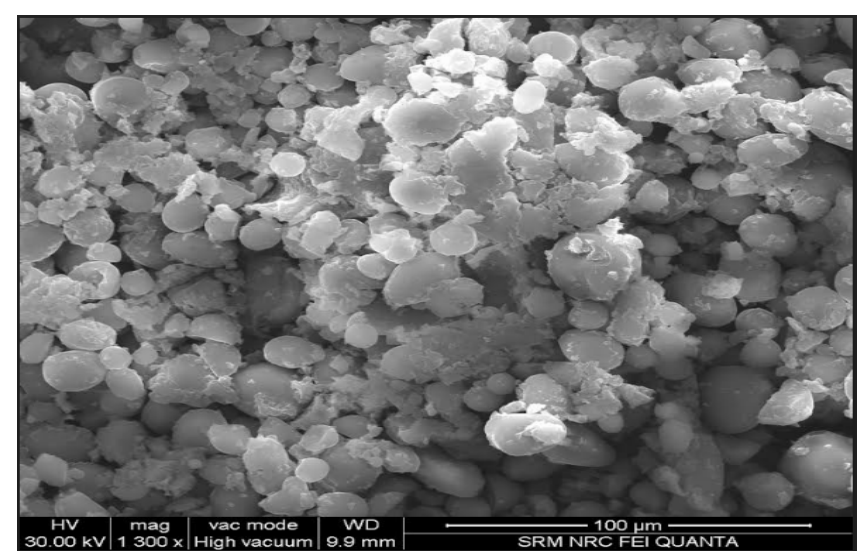

Fig. 6: Scanning electron microscopy of nimodipine SLN A4 (Containing tween 80, palmitic acid and drug concentration-100 mg)

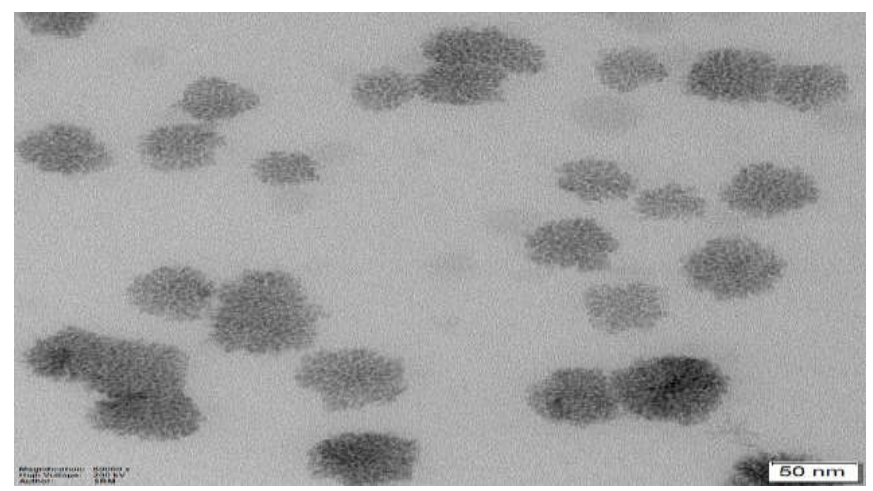

Fig. 7: Transmission electron microscopy of nimodipine SLN F4 (Containing Tween 80, stearic acid and drug concentration-100 mg) 


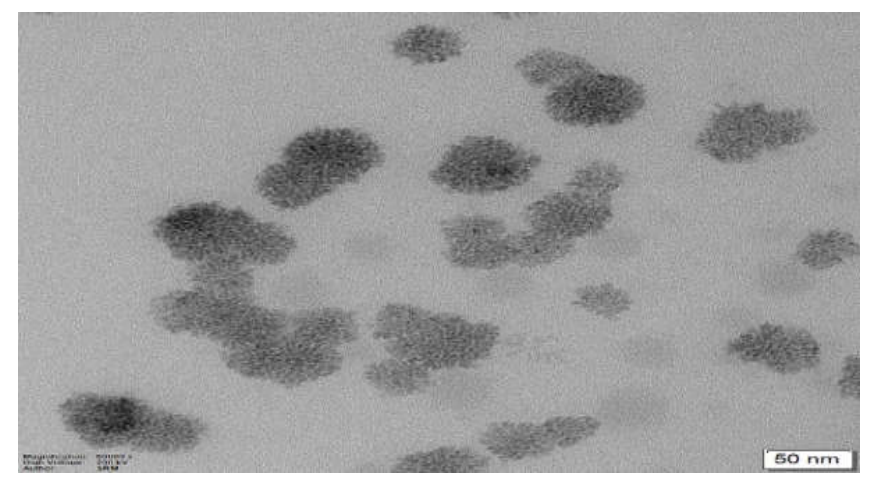

Fig. 8: Transmission electron microscopy of nimodipine SLN A4 (Containing tween 80, palmitic acid and drug concentration-100 mg)

\section{Stability studies}

For the samples with high entrapment efficiencies, stability studies were conducted. The temperatures maintained were $4{ }^{\circ} \mathrm{C}$ and 25 ${ }^{\circ} \mathrm{C} \pm 2{ }^{\circ} \mathrm{C}$ and the time for the study was $180 \mathrm{~d}$. Stability studies identified that the SLNs were physically stable.

\section{DISCUSSION}

Nimodipine solid lipid nanoparticles were prepared by hot homogenization technique followed by ultrasonication. The method was able to produce nanoparticles of an acceptable range. FT-IR spectroscopic studies were conducted to determine possible drug: lipid interactions. FT-IR spectrum of pure drug nimodipine, stearic acid, palmitic acid and physical mixture of nimodipine with stearic acid, palmitic acid tween 80 were obtained and shown in fig. 2 . The characteristic peaks of nimodipine and lipids were also present in the spectra of physical mixtures, thus indicating that there is no significant evidence of chemical interaction between drug and lipids The SLNs were characterized for average particle size, PDI, zeta potential and percentage drug entrapped efficiency. The formulation resulted in consistent nanoparticles of narrow size distribution and bring about a slight increase in particle sizes from $119.32 \pm 39.93$ to $138.71 \pm 30.95 \mathrm{~nm}$ for formulation $\mathrm{F} 1$ to $\mathrm{F} 6$ and $132 \mathrm{~nm}$ to $158 \mathrm{~nm}$ for formulation (A1-A6). These findings are in reliable with the study of Jensen et al. [21] who explained that the increase in size of SLNs after integration of drug replicated the dissolution of the drug in the lipid phase. A narrow particle size distribution was a sign of nanoparticles stability and homogeneous dispersion [22]. Zeta potential of various nimodipine loaded SLNs with stearic acid varies from-17.6 to-37.6 mV and the polydispersity index was found to be between 0.162 to 0.265 (table 4). Zeta potential of various nimodipine loaded SLNs with palmitic acid varies from-17.6 to-34 $\mathrm{mV}$ and polydispersity index was found to be between 0.155 to 0.275 (table 4). PDI values ranging from 0 to 0.5 were measured to be monodisperse and homogenous, but those of more than 0.5 indicated non-homogeneity and polydispersity [23]. In the present study, the particle size distribution was monodisperse and homogenous as formulation has less mean \pm standard error PDI of $0.17 \pm 0$. According to Schwarz and Mehnert [22] and Zimmermann et al. [24], the negative charge of zeta potential was deliberated by the lipids used in the SLNs. In a pact with this, the PA utilized in this study provided a negative charge of zeta potential. Nanoparticle with zeta potential values $>+25 \mathrm{mV}$ or $<-25 \mathrm{mV}$ usually has high degrees of stability due to electric repulsion between particles. Dispersions with a low zeta potential value will aggregate due to Van Der Waal inter-particle attraction [25]. In this study, the mean \pm SD zeta potential of $-14.2 \pm 1.00$. These SLNs of stearic acid (F1-F6) presented drug entrapment efficiency of $81.2 \%$ to $85.80 \%$ and that of palmitic acid (A1-A6) shown $76.8-81.8 \%$ which is due to its lipophilic nature (table 4). In vitro drug release study was executed for all the formulations SLN F1 to SLN F6 of 76.1 to 83\% and SLN A1 to SLN A6, which shows the sustained release of drug of about 78$85 \%$ respectively after $12 \mathrm{~h}$. The initial release should be sufficiently rapid to confirm that the therapeutic drug levels are attained in a timely manner in vivo. The subsequent slow release is mainly due to the slow diffusion of drug molecules through the lipid matrix of the nanoparticles [24, 25]. Slow drug release will contribute to maintaining the effective therapeutic drug concentrations. The optimized Nimodipine SLNs were preferred as SLNs F4 and A4 which shows a least zeta potential and good EE. As the concentration of surfactant mixture is increased exhibited smallest particle size and it has been found out that Tween 80 promotes the formation of smaller sized nanoparticles and were observed that increased content of surfactant supports the formation of smaller nanoparticles [25]. All the SLNs formulations showed negative zeta potential values, which indicate the stable nature of nanoparticles due to electrostatic repulsion. As the surfactant content increased, EE increases, which could be due to the development of stabilized nanoparticles. This increase in solubility is probably responsible for an increase in the drug release in acid medium and increased lipid content reduced the cumulative release, which is in consistent with the earlier reports. The lipid content increases the packing density of lipid molecules in given space as a result of release is reduced. For further studies, based on the particle size, PDI, zeta potential, and in vitro release, $\mathrm{F} 4$ and $\mathrm{A} 4$ was considered

\section{CONCLUSION}

In this work, the Nimodipine drug was successfully loaded into SLNs by modified high shear homogenization and ultra-sonication techniques. The effects of different formulation variables on percentage entrapment efficiency and physiochemical properties were evaluated. The in vitro release tests showed that the slow release of drug release. The type and concentration of surfactant, as well as the lipid matrix, were played a greater role on physicochemical characterization of SLNs and the in vitro drug release. SLN formulation F4 and A4 composed of Tween 80 as a surfactant with higher concentration and the lower concentration of lipid matrix $(1000 \mathrm{mg}$ of stearic acid for F4 and $1000 \mathrm{mg}$ of palmitic acid for A4) showed the best result in view of the entrapment efficiency as well as in vitro drug release. Particle size analysis showed that the formed particles were in Nano size. Based on observation, it can be concluded that the formulated lipid Nanoparticulate delivery system of Nimodipine could be widely accepted and physiologically safe lipids were capable of exhibiting sustained properties. In conclusion, the SLNs designed in this work were shown efficient drug delivery systems for poor soluble drugs like nimodipine.

\section{FUNDING}

$\mathrm{Nil}$

\section{AUTHORS CONTRIBUTIONS}

All the authors have contributed equally.

\section{CONFLICT OF INTERESTS}

Authors declare no conflict of interest amongst themselves.

\section{REFERENCES}

1. Akter M, Banik S, Hossain MS. In vitro evaluation of oral extended-release drug delivery system for metoprolol succinate using kollidon SR. J Appl Pharm Sci 2012;25:188-92. 
2. Muller RH, Maeder K, Gohla S. Solid lipid nanoparticles (SLN) for controlled drug delivery-a review of state of the art. Eur J Pharm Biopharm 2000;50:161-77.

3. Kaur IP, Singh H. Nanostructured drug delivery for better management of tuberculosis. J Controlled Release 2014;184:36-50.

4. Singh S, Dobhal AK, Jain A, Pandit JK, Chakraborty S Formulation and evaluation of solid lipid nanoparticles of a water-soluble drug: zidovudine. Chem Pharm Bull 2010;58:650-5.

5. Svilenov H, Tzachev C. Solid lipid nanoparticles-a promising drug delivery system. Nanomedicine 2014;2:187-237.

6. Gastaldi L, Battaglia L, Peira E, Chirio D, Muntoni E, Solazzi I, et al. Solid lipid nanoparticles as vehicles of drugs to the brain: current state of the art. Eur J Pharm Biopharm 2014;87:433-44.

7. Ekambaram P, Sathali AA, Priyanka K. Solid lipid nanoparticles: a review. Sci Rev Chem Commun 2012;2:80-102.

8. Yadav NE, Khatak SU, Sara US. Solid lipid nanoparticles-a review. Int J Appl Pharm 2013;5:8-18.

9. Basu T, Pal B, Singh S. Hollow chitosan nanocomposite as drug carrier system for controlled delivery of nimodipine. Chem Phys Lett 2018;706:465-71.

10. Shafiq-un-Nabi S, Shakeel F, Talegaonkar S, Ali J, Baboota S, Ahuja A, et al. Formulation development and optimization using nanoemulsion technique: a technical note. AAPS PharmSciTech 2007;8:12-7.

11. Sreelola VU, Sailaja AK, Pharmacy M. Preparation and characterisation of ibuprofen-loaded polymeric nanoparticles by a solvent evaporation technique. Int J Pharm Pharm Sci 2014;6:416-21.

12. Hou D, Xie C, Huang $\mathrm{K}$, Zhu C. The production and characteristics of solid lipid nanoparticles (SLNs). Biomaterials 2003;24:1781-5.

13. Mehnert W, Mäder K. Solid lipid nanoparticles: production, characterization and applications. Adv Drug Delivery Rev 2012;64:83-101

14. Dubes A, Parrot Lopez H, Abdelwahed W, Degobert G, Fessi H, Shahgaldian $\mathrm{P}$, et al. Scanning electron microscopy and atomic force microscopy imaging of solid lipid nanoparticles derived from amphiphilic cyclodextrins. Eur J Pharm Biopharm 2003;55:279-82

15. Shafiq S, Shakeel F, Talegaonkar S, Ahmad FJ, Khar RK, Ali M. Development and bioavailability assessment of nimodipine nanoemulsion formulation. Eur J Pharm Biopharm 2007;66:227-43.

16. Senthil Kumar P, Arivuchelvan A, Jagadeeswaran A, Subramanian N. Formulation, optimization and evaluation of enrofloxacin solid lipid nanoparticles for sustained oral delivery. Asian J Pharm Clin Res 2015;8:231-6.

17. Poovi Ganesan, Damodharan Narayanasamy. Lipid nanoparticles: different preparation techniques, characterization, hurdles, and strategies for the production of solid lipid nanoparticles and nanostructured lipid carriers for oral drug delivery. Sustainable Chem Pharm 2017;6:37-56.

18. Ekambaram P, Sathali AA. Formulation and evaluation of solid lipid nanoparticles of ramipril. J Young Pharma 2011;3:216-20.

19. Remya PN, Damodharan. N formulation, development, and characterization of cilnidipine loaded solid lipid nanoparticles Asian J Pharm Clin Res 2018;11:120-5.

20. Marwah H, Garg T, Goyal AK, Rath G. Permeation enhancer strategies in transdermal drug delivery. Drug Delivery 2016;23:564-78.

21. Jensen LB, Magnussson E, Gunnarsson L, Vermehren C, Nielsen HM, Petersson K. Corticosteroid solubility and lipid polarity control release from solid lipid nanoparticles. Int I Pharm 2010;390:53-60

22. Schwarz C, Mehnert W. Solid lipid nanoparticles (SLN) for controlled drug delivery. II. Drug incorporation and physicochemical characterization. J Microencapsul 1999;16:205-13.

23. Tiyaboonchai W, Tungpradit W, Plianbangchang P. Formulation and characterization of curcuminoids loaded solid lipid nanoparticles. Int J Pharm 2007;337:299-306.

24. Zimmermann E, Müller RH, Mader K. Influence of different parameters on reconstitution of lyophilized SLN. Int J Pharm 2000;196:211-3.

25. Havanoor SM. Isradipine loaded solid lipid nanoparticles for better treatment of hypertension-preparation, characterization and in vivo evaluation. Int J Biopharm 2014;5:218-24. 